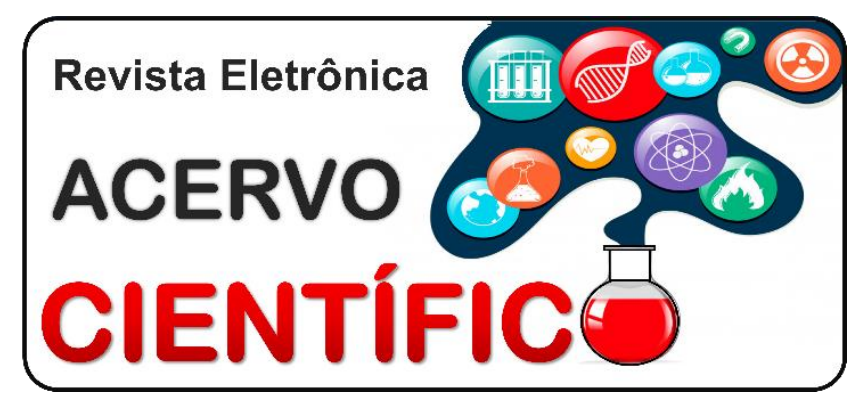

\section{REVISÃO BIBLIOGRÁFICA}

Recebido em: 11/2020

Aceito em: 12/2020

Publicado em: 1/2021

\title{
Desafios dos profissionais da atenção básica em relação à polifarmácia e à polimorbidade em idosos
}

\author{
Challenges of primary care professionals regarding polypharmacy and polymorbidity in the \\ elderly
}
Desafíos de los profesionales de atención primaria en relación con la polifarmacia y la polimobilidad en los ancianos

Layra Ton ${ }^{1 *}$, Mônica Isaura Corrêa ${ }^{2}$, Gabriela Benedini Strini Portinari Beja ${ }^{3}$, Isabella Doriguêto Moreira $^{4}$, Maria Paula Ferreira Soares ${ }^{5}$, Mariana Moreira Penedo ${ }^{6}$, Paula Medina Cribari ${ }^{4}$, Renan Pinheiro Laignier ${ }^{1}$, Samara Lopes Mendes ${ }^{4}$, Victor Cabreira Frazão ${ }^{7}$.

\begin{abstract}
Resumo: Este artigo trata-se de uma revisão narrativa que buscou discutir a polifarmácia e os desafios na assistência aos idosos que apresentam duas ou mais doenças crônicas não transmissíveis (DCNT), como hipertensão arterial, diabetes, cardiopatias e doenças de caráter reumático, caracterizando o quadro de polimorbidade. Além disso, abordou a formação de uma equipe multidisciplinar com conhecimento em geriatria como maneira de auxiliar o autocuidado e diminuir a polimorbidade e a polifarmácia, possibilitando uma melhoria na qualidade de vida para os idosos. Assim sendo, observa-se que a falta de interação entre os níveis de saúde e a assistência média esporádica resultam em um mau prognóstico para o paciente. Ainda há de considerar-se que a polifarmácia traz uma associação de medicamentos que pode causar reações adversas e sintomas que apontem para algum tipo de enfermidade, mas que são fruto do uso indiscriminado de medicação, exigindo que novos medicamentos sejam associados para controle dos sintomas encontrados e piorando o quadro de saúde do paciente. Por fim, nota-se que a organização social do trabalho do profissional de saúde em concomitância com as limitações na área de atuação dos profissionais de saúde propicia uma baixa qualidade de vida durante o processo de senescência.
\end{abstract}

Palavras-chave: Atenção Primária à Saúde, Morbidade, Polifarmácia.

Abstract: This article is a narrative review that sought to discuss the polypharmacy and the challenges in assisting the elderly who have two or more chronic noncommunicable diseases (CNCDs), such as high blood pressure, diabetes, heart disease and rheumatic diseases, characterizing the polymorbidity. In addition, it addressed the formation of a multidisciplinary team with knowledge in geriatrics as a way to assist self-care

\footnotetext{
${ }^{1}$ Centro Universitário de Caratinga (UNEC), Caratinga - MG. *E-mail: tonlayra@hotmail.com

${ }^{2}$ Faculdade de Medicina do Vale do Aço (FAMEVAÇO), Ipatinga - MG.

${ }^{3}$ Centro Universitário Barão de Mauá (CBM), Ribeirão Preto - SP.

${ }^{4}$ Centro Universitário UNIFAMINAS (UNIFAMINAS), Muriaé - MG.

${ }^{5}$ Centro Universitário Ingá (UNINGÁ), Maringá - PR.

${ }^{6}$ Universidade de Vassouras (UV), Vassouras - RJ.

7 Faculdade de Medicina do Centro Universitário de Belo Horizonte (UniBh), Belo Horizonte - MG.
} 
and decrease polymorbidity and polypharmacy, enabling an improvement in the quality of life for the elderly. Thus, it is observed that the lack of interaction between health levels and sporadic average care results in a poor prognosis for the patient. It should also be considered that polypharmacy brings an association of drugs that can cause adverse reactions and symptoms that point to some type of illness, but that are the result of indiscriminate use of medication, requiring that new drugs be associated to control the symptoms found and worsening the patient's health status. Finally, it is noted that the social organization of the work of the health professional in conjunction with the limitations of health professionals performance provides a low quality of life during the senescence process.

Keywords: Primary Health Care, Morbidity, Polypharmacy.

Resúmen: Este artículo es uma revisión narrativa que buscó discutir la polifarmacia y los desafíos en la asistencia a los ancianos que padecen dos o más enfermedades crónicas no transmisibles (ECNT), como hipertensión arterial, diabetes, cardiopatías y enfermedades reumáticas, caracterizando la polimorbilidad. Además, abordó la formación de un equipo multidisciplinar con conocimientos en geriatría como una forma de ayudar al autocuidado y disminuir la polimorbilidad y polifarmacia, posibilitando una mejora en la calidad de vida de las personas mayores. Así, se observa que la falta de interacción entre los niveles de salud y la atención media esporádica da como resultado un mal pronóstico para el paciente. También se debe considerar que la polifarmacia trae consigo una asociación de fármacos que pueden provocar reacciones adversas y síntomas que apuntan a algún tipo de enfermedad, pero que son el resultado del uso indiscriminado de medicamentos, requiriendo que se asocien nuevos fármacos para controlar los síntomas encontrados. y empeoramiento del estado de salud del paciente. Finalmente, se observa que la organización social del trabajo del profesional de la salud en conjunto con las limitaciones en el área de actuación de los profesionales de la salud, proporciona una baja calidad de vida durante el proceso de senescencia.

Palabras clave: Atención Primaria de Salud, Morbilidad, Polifarmacia.

\section{INTRODUÇÃO}

A expectativa de vida tem aumentado progressivamente na população, proporcionando uma maior taxa de envelhecimento de maneira acelerada. No Brasil, esse processo de senescência associa-se, muitas vezes, ao aumento da prevalência e da incidência de doenças crônicas não transmissíveis (DCNT), como hipertensão e diabetes, as quais acometem mais de $70 \%$ dos brasileiros com mais de 60 anos (REGO AS, et al., 2017).

Além disso, um estudo transversal de base populacional, por meio de entrevistas com idosos, evidenciou entre zero e oito doenças presentes de forma variada nesses indivíduos, sendo que aproximadamente $7 \%$ apresentaram mais de quatro DCNT, como hipertensão arterial, diabetes, cardiopatias e doenças reumáticas. Nessa perspectiva, constata-se que os idosos podem dispor de mais de uma condição patológica simultaneamente, levando ao quadro de polimorbidade, ou seja, presença concomitante de doenças crônicas (RAMOS LR, et al., 2016).

Ademais, o enfrentamento das polimorbidades pelos idosos, na maioria dos casos, torna-os adeptos à terapia polifarmacológica, a qual se trata do uso de pelo menos cinco medicamentos. Notavelmente, apesar da administração correta desses polifármacos ser imprescindível, isso não tem sido feito de maneira efetiva e satisfatória (RAMOS LF, et al., 2016). Assim, torna-se evidente que o uso de vários fármacos ao mesmo tempo pode ocasionar interações medicamentosas indesejadas e dificultar a administração posológica e a gestão dos medicamentos, tornando esses pacientes mais suscetíveis à morbimortalidade, ao risco de quedas, às novas internações e aos gastos excessivos, constituindo um problema de saúde pública (CORONADO VV, et al., 2019; ROCHON PA, et al., 2020).

Nessa perspectiva, a Organização Mundial da Saúde (OMS) reconhece a importância de estabelecer uma política nacional de medicamentos, com o objetivo de um uso racional dos medicamentos, trazendo maiores benefícios ao paciente. Dessa forma, no intuito de melhor atendimento à saúde do idoso, o Ministério da Saúde brasileiro criou a Política Nacional de Saúde à Pessoa Idosa, visando à promoção, à prevenção e à 
recuperação da saúde por meio da Atenção Primária à Saúde (APS) (REGO AS, et al., 2017; CHIAPELLA LC, et al., 2018).

Nesse contexto, sabe-se que a APS, dispondo-se de uma equipe multiprofissional articulada, deveria contribuir positivamente para o acolhimento e o tratamento polifarmacológico dos idosos com polimorbidades. No entanto, esses profissionais têm enfrentado dificuldades significativas para colocar em prática o que as políticas nacionais defendem, devido, muitas vezes, à baixa implementação estrutural da APS, à resistência do paciente em seguir as orientações médicas, à má relação entre paciente e equipe multiprofissional e à pouca aderência ao tratamento e às orientações por parte do idoso (KÖBERLEIN-NEU J, et al., 2016; PÉREZJOVER V, et al., 2018).

Diante disso, o presente artigo tem o objetivo de revisar e identificar os obstáculos enfrentados pelos profissionais da atenção básica em relação ao gerenciamento e ao manejo da polifarmácia e da polimorbidade em idosos, e ratificar a importância do acolhimento contínuo e integral desses indivíduos ao nível de atenção primária à saúde.

\section{REVISÃO BIBLIOGRÁFICA}

O século XXI passa pelo envelhecimento populacional. O crescimento da população idosa mundial é em torno de $3 \%$ ao ano, e para o ano de 2050 estima-se que os habitantes idosos serão de 2,1 bilhões de pessoas (SOUSA NFS, et al., 2018). Da mesma forma, o Brasil passa por um crescente processo de aceleração de envelhecimento populacional, de acordo com dados fornecidos pela OMS, até o ano de 2025 o Brasil deverá possuir a sexta maior população idosa do mundo, com aproximadamente 32 milhões de pessoas em idade superior a 60 anos, significando 13\% da população Brasileira (RAMOS LR, et al., 2016).

Diante disso, Amaral TLM, et al. (2016) afirma que no envelhecimento ocorre um processo de mudanças, as quais vão desde o nível molecular até o morfofisiológico, propiciando uma diminuição natural de capacidades corporais. Nesse contexto, devido à queda de função fisiológica do corpo, processo natural da senescência, os idosos são mais suscetíveis ao acometimento por DCNT, sendo as principais relacionadas ao sistema cardiovascular, como hipertensão arterial sistêmica, acidente vascular encefálico, aumento na glicemia podendo resultar em diabetes mellitus, câncer e depressão (LEITE BC, et al., 2019; AMARAL TLM, et al., 2016).

Ademais, Mari FR, et al. (2016) informam que múltiplas doenças crônicas podem acometer um único indivíduo, o que corrobora a presença de multimorbidade entre os idosos. Assim, a multimorbidade é conceituada pela presença de duas ou mais doenças crônicas físicas ou mentais em um indivíduo. Notavelmente, as pessoas que se encontram nessa situação, principalmente idosos, são predispostas a passarem por um maior número de hospitalizações, utilizando conjuntamente muitos medicamentos e, assim, aumentando a vulnerabilidade aos efeitos adversos (CAVALCANTI G, et al., 2017).

Dessa forma, Nunes BP, et al. (2017), a partir de um estudo no Brasil, revelam que aproximadamente $50 \%$ a $98 \%$ da população idosa possui multimorbidade com tendência a aumentar com o avançar da idade. Sendo assim, os profissionais do Sistema Único de Saúde (SUS) enfrentam desafios no manejo adequado desses indivíduos, o que torna necessário maior planejamento e articulação de ações para a efetivação do atendimento dessa demanda (NUNES BP, et al., 2018). Nessa perspectiva, acredita-se que a multimorbidade é um dos principais problemas de saúde pública no mundo, atingindo países desenvolvidos, em desenvolvimento ou subdesenvolvidos, implicando em elevados índices de morbimortalidades (CARVALHO JN, 2017).

Com isso, essa mudança na feição epidemiológica do mundo e no Brasil causa impacto negativo nos cofres públicos, devido às despesas com tratamentos médicos e hospitalares. Ademais, as doenças dos idosos, muitas vezes, por serem crônicas e múltiplas, perduram por vários anos e exigem acompanhamento integral e intervenções contínuas pelos profissionais da atenção básica (MIRANDA GMD, et al., 2017).

Além disso, Veras RP e Oliveira M (2018) afirmam que esses profissionais passam por desafios, visto que a formação de uma equipe multidisciplinar com conhecimento em geriatria é importante, pois auxilia os idosos 
no âmbito do autocuidado para com a polimorbidade e a polifarmácia, possibilitando melhoria da qualidade de vida dos mesmos.

Assim, sabe-se que a prescrição de múltiplos fármacos em geriatria é uma rotina nos ambulatórios da Atenção Básica à Saúde. Ademais, a polifarmácia em idosos pode ser considerada um problema quando prescrita erroneamente, principalmente, em pessoas com baixos níveis de escolaridade e socioeconômicos. Em contrapartida, quando bem administrada tanto pelo profissional da saúde quanto pelo paciente, a polifarmácia é considerada positiva. No entanto, nota-se que nem sempre isso acontece, uma vez que o sistema de saúde, os profissionais e os pacientes convivem com diversos empecilhos cotidianos, os quais impedem a plena efetivação do uso correto de vários fármacos simultaneamente em pacientes com múltiplas morbidades; neste caso, a saber, os idosos. Assim, não só a interação medicamentosa negativa, mas também o uso desses fármacos de maneira errada propiciam consequências nocivas para o bem-estar do paciente, como declínio da funcionalidade do organismo, perda de memória, distúrbios do sono e humor, palpitações, quedas, depressão, vertigem, tontura, sonolência e agitação, ocasionando uma baixa qualidade de vida (SÁNCHES-RODRÍGUEZ JR, et al., 2019).

Nesse contexto, considera-se que a polifarmácia no idoso proporciona repercussões quantitativas e qualitativas. Dessa maneira, percebe-se que ela pode estar associada somente à utilização de várias medicações concomitantemente, podendo ocasionar interações indesejáveis. Além disso, tem-se também o aspecto qualitativo, o qual está relacionado à eficácia do uso do medicamento e à adequação da prescrição. Logo, apesar da quantidade de fármacos prescrita em consultas geriátricas estar diretamente relacionada com as doenças crônicas apresentadas pelo paciente, torna-se um desafio quando não aderida da forma correta por esse idoso ou mal administrada pelo profissional da saúde, o que acontece, muitas vezes, tanto pela falta de capacitação técnica quanto pela precária estrutura fornecida pelo sistema de saúde (HSIANGWEN L, 2018).

Nessa perspectiva, entende-se que a equipe profissional de saúde é responsável pelo monitoramento e gerenciamento das prescrições medicamentosas, o que, em um cenário ideal, deve ser realizado de maneira consciente conforme a necessidade particular de cada paciente, visando ao desenvolvimento de uma prática clínica que efetiva o papel desses profissionais na prevenção de incidentes relacionados com a segurança do tratamento e de iatrogenias (CORONADO-VÁQUEZ V, et al., 2019).

A título de exemplificação, a Agency for Healthcare Reserarch and Quality (AHRQ) dos Estados Unidos da América (EUA) conduz a atuação desses profissionais com a finalidade de direcionar uma prescrição segura, oferecendo ferramentas de auxílio que ajudam os pacientes a autogerenciarem seu cuidado de maneira mais eficaz em relação ao seu tratamento, consolidando-o. Assim, no ambiente da Atenção Básica, os recursos são direcionados para fomentar uma participação ativa desses idosos no curso de sua terapêutica, por meio da promoção de orientações para uso seguro de medicações, como anticoagulantes, e da apresentação de mecanismos que visam melhorar a comunicação entre o médico e o paciente (CORONADO-VÁQUEZ V, et al., 2019).

Além disso, sabe-se que a senescência, apesar de ser um processo fisiológico, não acontece sempre de maneira homogênea, visto que cada organismo possui suas particularidades durante o envelhecimento (RIBEIRO IA, et al., 2018). Dessa forma, com o envelhecimento populacional acelerado, as políticas públicas relacionadas aos idosos têm ganhado notoriedade no âmbito da saúde. Ademais, essas medidas governamentais têm em vista a promoção, a prevenção e a manutenção da saúde, principalmente, por meio da APS; contudo, percebe-se que tais medidas não têm sido efetivadas em sua plenitude, tornando-se um desafio a ser superado na atuação dos profissionais da saúde (DAMACENO MJCE e CHORELLI MQ, 2019).

Neste contexto, o Ministério da Saúde brasileiro implementou a Política Nacional de Saúde da Pessoa Idosa (PNSPI), a qual foi aprovada pela portaria MS n. 2.528/2006. Diante disso, a PNSPI tem a finalidade de restabelecer, manter e estimular a autonomia desta classe, na qual, são considerados idosos os indivíduos faixa etária maior de 60 anos, destinando medidas coletivas e individuais de saúde (REGO, et al., 2017). Além disso, Damaceno MJCF e Chirelli MQ (2019) analisaram a implementação das ações da PNSPI na Estratégia da Saúde da Família e, a partir disso, notaram que, apesar de existir políticas em prol do 
gerenciamento da saúde da pessoa idosa, seu desempenho ainda não é satisfatório conforme a metodologia proposta. Notavelmente, esse cenário ocorre, muitas vezes, devido à dificuldade enfrentada pelos municípios na realocação de recursos financeiros e ao baixo fornecimento de estrutura e de recursos para a atuação adequada e capacitada dos profissionais da saúde na APS (SALDIVA PHN e VERAS M, 2018).

Dessa forma, por mais que a PNSPI seja promissora no papel, ainda não tem sido suficiente para a promoção plena de saúde aos idosos, uma vez que ainda não suprime as adversidades apresentadas no cotidiano desses pacientes, como falta de conhecimento sobre a necessidade de consultas regulares e problemas de acessibilidade ao sistema, por exemplo, devido à falta de acompanhante e de serviços de transporte de maneira menos onerosa. Assim, esse indivíduo não efetiva seu processo de cuidado na atenção primária, necessitando de serviços tanto na atenção secundária quanto terciária, o que propicia a fragmentação entre os setores e impossibilita uma comunicação e uma política eficiente (COELHO LP, et al., 2018).

Notavelmente, o processo de envelhecimento populacional não ocorre somente no Brasil, mas também nos países desenvolvidos. Além disso, a efetivação do completo bem-estar físico, social e mental exige o trabalho conjunto entre a medicina, o governo e a sociedade (PEREIRA AG e ALVES LC, 2016). Diante disso, a título de exemplificação, o sistema de saúde de Gana, África, é um dos sistemas mais bem desenvolvidos e modelo para outros países, pois conta com uma cobertura universal de saúde (WHO, 2015). No entanto, enfrentam dificuldades para que o acesso seja possibilitado a todos, levando à necessidade da parcela não atendida a recorrer à automedicação (PEREIRA AG e ALVES LC, 2016).

Ainda nesse contexto, o financiamento em saúde é um fator relevante na África e ocorre pelo The National Health Insurance Scheme (NHIS), o qual visa reduzir despesas e riscos com a população; porém, ainda existem desincentivos financeiros para sua melhor implementação. Com isso, estabeleceram como método alternativo a alfabetização em saúde (AS), a qual propicia melhor capacitação, autocuidado e autogerenciamento para auxiliar os indivíduos em seu cuidado de maneira integral e eficaz (PEREIRA AG e ALVES LC, 2016).

Nesse contexto, há muitos desafios a serem abordados na atenção básica para que se obtenha a segurança do paciente idoso frente à polifarmácia e à polimorbidade. O processo de tornar-se senil pode levar a alterações na farmacodinâmica e farmacocinética dos medicamentos, o que propicia interações medicamentosas, reações adversas e toxicidade. Sendo assim, é necessário conhecer as formas de manifestação da doença no idoso, para que sua apresentação não seja confundida com o processo fisiológico de senescência. O contrário também pode ocorrer, quando reações adversas podem ser trocadas com doenças, acarretando na prescrição de novos fármacos, o que acaba atingindo um processo iatrogênico e criando um efeito cascata, ou seja, uma droga fornece efeito indesejado quando associada a outra, aumentando o risco de interação medicamentosa (SILVA EMA e AGUIAR RS, 2020; RODRíGUEZ JRS, et al., 2018).

Ademais, sabe-se que os idosos necessitam de um olhar holístico para a elaboração de um adequado esquema terapêutico, sendo uma questão imprescindível para realização de um trabalho interdisciplinar e multiprofissional. Quando não recebem adequada assistência psicossocial e médica, acaba resultando em internações mais prolongadas, o que deixa sua capacidade funcional debilitada, afetando a habilidade física e cognitiva, a mobilidade e o equilíbrio dos parâmetros hemodinâmicos (SILVA EMA e AGUIAR RS, 2020; RODRÍGUEZ JRS, et al.,2018).

Além disso, outro problema existente é a automedicação. Muitos pacientes se diagnosticam sozinhos, sem ir a um consultório médico e isso acaba originando interações com o tratamento correto. Essa questão está muito relacionada ao fator socioeducacional, em que quanto menor o nível educacional e social, maior o consumo de medicamentos sem receita, o que proporciona a não adesão do paciente em um tratamento correto (RODRÍGUEZ JRS, et al., 2018). Nessa perspectiva, outra adversidade dos profissionais de saúde é a incapacidade aliada à baixa experiência, assim como a falta de integração entre os níveis de saúde, somada à policonsulta e a uma assistência médica não programada, o que promove um mau prognóstico para o paciente, uma vez que a atuação efetiva desse profissional é dependente de muitos fatores, como os 
descritos. Assim, evidencia-se a necessidade de melhoria na efetivação dos protocolos clínicos, além da maior qualificação dos profissionais (SILVA EMA e AGUIAR RS, 2020; RODRíGUEZ JRS, et al., 2018).

\section{CONSIDERAÇÕES FINAIS}

Com base neste estudo, constata-se que a população idosa vem aumentando progressivamente em nível mundial. Assim, têm-se cada vez mais novos desafios, como a polimorbidade, a polifarmácia e suas repercussões. Neste contexto, nota-se que as múltiplas adversidades, as quais vão desde a baixa aderência ao tratamento por parte do paciente à falta de estrutura e suporte social para o acompanhamento do idoso, propiciam baixa eficácia no tratamento geriátrico em seu nível multidisciplinar e manejo minucioso, impedindo o melhor desenvolvimento em relação ao tratamento. Logo, é indispensável possibilitar maior promoção de saúde para esses indivíduos, em que, por meio de planos governamentais resolutivos, os profissionais de saúde possam efetivar uma melhor conduta para esse paciente, promovendo um atendimento ao idoso eficaz. Dessa maneira, é propiciado ao processo de senescência desses indivíduos maior qualidade de vida, bemestar físico e psíquico, superação dos obstáculos e acolhimento integral.

\section{REFERÊNCIAS}

1. AMARAL TLM, et al. Multimorbidade, depressão e qualidade de vida em idosos atendidos pela Estratégia de Saúde da Família em Senador Guiomard, Acre, Brasil. Ciência e Saúde Coletiva, 2018; 23(9):3077-3084.

2. CARVALHO JN. Epidemiologia da Multimorbidade na População Brasileira. Tese de Doutorado. Universidade Federal do Rio Grande do Norte, 2017.

3. CAVALCANTI G, et al. Multimorbidade associado à polifarmácia e autopercepção negativa de saúde. Revista Brasileira de Geriatria e Gerontologia, 2017; 20(5): 635-643.

4. CHIAPELLA LC, et al. Potentially Inappropriate Medications in Elderly Ambulatory Patients: A Comparative Study between a Primary Health Care Center and a Community Pharmacy. Value in Health Regional Issues, 2018; 17: 119125.

5. COELHO LP, et al. Rede de atenção ao idoso: fatores facilitadores e barreiras para implementação. Revista de Saúde Coletiva, 2018; 28(4):1-19.

6. CORONADO-VÁQUEZ V, et al. Shared Decision-Making in Chronic Patients with Polypharmacy: An Interventional Study for Assessing Medication Appropriateness. Journal Of Clinical Medicine, 2019; 8 (6): 904-917.

7. DAMACENO MJCF, CHIRELLI MQ. implementação da Saúde do idoso na estratégia Saúde da Família: visão dos profissionais e gestores. Ciência \& Saúde Coletiva, 2019; 24(5):1637-1646.

8. HSIANG-WEN L, et al. Economic outcomes of pharmacist-physician medication therapy management for polypharmacy elderly: A prospective, randomized, controlled trial. Journal of the Formosan Medical Association, 2018; 117: 235-243.

9. KÖBERLEIN-NEU JK, et al. Interprofessional Medication Management in Patients With Multiple Morbidities: A Cluster-randomized Trial (the WestGem Study). Deutsches Ärzteblatt International, 2016; 113: 741-748.

10. LEITE BC, et al. Multimorbidade por doenças crônicas não transmissíveis em idosos: estudo de base populacional. Revista Brasileira de Geriatria e Gerontologia, 2019; 22(6): 190253.

11. MARI FR, et al. O processo de envelhecimento e a saúde: o que pensam as pessoas de meia idade sobre o tema. Revista Brasileira de Geriatria e Gerontologia, 2016; 19(1):35-44.

12. MIRANDA GMD, et al. Desafios das políticas públicas no cenário de transição demográfica e mudanças sociais no Brasil. Interface (Botucatu), 2017; 21(61):309-20.

13. NUNES BP, et al. Hospitalização em idosos: associação com multimorbidade, atenção básica e plano de saúde. Revista de Saúde Pública, 2017; 51:43.

14. NUNES BP, et al. Multimorbidade em indivíduos com 50 anos ou mais de idade: ELSI-Brasil. Revista de Saúde Pública, 2018; 52 (Supl 2):10s.

15. PEREIRA AG, ALVES LC. Condição de vida e saúde dos idosos: uma revisão bibliográfica. Textos NEPO (UNICAMP), 2016; 75:1-27.

16. PÉREZ-JOVER V, et al. Inappropriate Use of Medication by Elderly, Polymedicated, or Multipathological Patients with Chronic Diseases. International Journal Of Environmental Research and Public Health, 2018; 15 (2): $310-324$.

17. RAMOS LR, et al. Polifarmácia e polimorbidade em idosos no Brasil: um desafio em saúde pública. Rev Saúde Pública, 2016; 50 (2): 1-9. 
18. REGO AS, et al. Fatores associados ao atendimento a idosos por condições sensíveis à Atenção Primária à Saúde. Rev. Bras. Geriatr. Gerontol., Rio de Janeiro, 2017; 20 (6): 778-789.

19. RIBEIRO IA, et al. Síndrome do idoso frágil em idosos com doenças crônicas na Atenção Primária. Revista da Escola de Enfermagem da USP, 2019; 53.

20. ROCHON PA, et al. Drug prescribing for older adults. Wolters Kluwer, 2020.

21. RODRÍGUEZ JRS, et al. Polifarmacia em adulto mayor, impacto em su calidad de vida. Revision de literatura. Revista de Salud Pública, 2019; 21(2); 271-277.

22. SÁNCHEZ-RODRíGUEZ JR, et al. Polifarmacia em adulto mayor, impacto em Su calidad de vida. Revision de literatura. Rev. Salud Pública, 2019; 21 (2): 271-277.

23. SALDIVA PHN, VERAS M. Gastos públicos com saúde: breve histórico, situação atual e perspectivas futuras. Estudos Avançados, 2018; 32(92).

24. SILVA EMA, AGUIAR RS. Fatores relacionados à polimedicação em idosos e a segurança do paciente: uma revisão integrativa. Revista Nursing, 2020; 23(265); 4127-4133

25. SOUSA NFS, et al. Envelhecimento ativo: prevalência e diferenças de gênero e idade em estudo de base populacional. Caderno de Saúde Pública, 2018; 34(11): e 00173317.

26. VERAS RP, OLIVEIRA M. Envelhecer no Brasil: a construção de um modelo de cuidado. Ciência e Saúde Coletiva, 2018; 23(6):1929-1936.

27. WHO. Health in 2015: from Millienium Development Goals (MDGs) to SDGs, Sustainable Development Goals. Geneva: World Health Organization (WHO) 2015. 